\title{
Article
}

\section{When distraction helps: Evidence that concurrent articulation and irrelevant speech can facilitate insight problem solving}

Ball, Linden, Marsh, John Everett, Litchfield, Damien, Cook, Rebecca and Booth, Natalie

Available at http://clok.uclan.ac.uk/13978/

Ball, Linden ORCID: 0000-0002-5099-0124, Marsh, John Everett ORCID: 00000002-9494-1287, Litchfield, Damien, Cook, Rebecca and Booth, Natalie (2015) When distraction helps: Evidence that concurrent articulation and irrelevant speech can facilitate insight problem solving. Thinking \& Reasoning, 21 (1). pp. 76-96. ISSN 1354-6783

It is advisable to refer to the publisher's version if you intend to cite from the work. http://dx.doi.org/10.1080/13546783.2014.934399

For more information about UCLan's research in this area go to http://www.uclan.ac.uk/researchgroups/ and search for <name of research Group>.

For information about Research generally at UCLan please go to http://www.uclan.ac.uk/research/

All outputs in CLoK are protected by Intellectual Property Rights law, including Copyright law. Copyright, IPR and Moral Rights for the works on this site are retained by the individual authors and/or other copyright owners. Terms and conditions for use of this material are defined in the policies page. 
RUNNING HEAD: DISTRACTION FACILITATES INSIGHT PROBLEM SOLVING

Final author draft only. The copyedited article may differ from this manuscript version. The details of the article are as follows:

Ball, L. J., Marsh, J. E., Litchfield, D., Cook, R. L., \& Booth, N. When distraction helps: Evidence that concurrent articulation and irrelevant speech can facilitate insight problem solving. Thinking \& Reasoning.

When Distraction Helps: Evidence that Concurrent Articulation and Irrelevant Speech Can Facilitate Insight Problem Solving

\author{
Linden J. Ball*, John E. Marsh
}

School of Psychology, University of Central Lancashire, UK

Damien Litchfield

School of Psychology, Edge Hill University, UK

Rebecca L. Cook, Natalie Booth

Department of Psychology, Lancaster University, UK

*Corresponding author:

Linden J. Ball

School of Psychology

University of Central Lancashire

Preston

Lancashire PR2 8BX, UK

Tel: +44 (0)1772 893421

Email: LBall@uclan.ac.uk 


\begin{abstract}
We report an experiment investigating the "special-process" theory of insight problem solving, which claims that insight arises from non-conscious, non-reportable processes that enable problem re-structuring. We predicted that reducing opportunities for speech-based processing during insight problem solving should permit special processes to function more effectively and gain conscious awareness, thereby facilitating insight. We distracted speechbased processing by using either articulatory suppression or irrelevant speech, with findings for these conditions supporting the predicted insight facilitation effect relative to silent working or thinking aloud. The latter condition was included to investigate the currently contested effect of "verbal overshadowing" on insight, whereby thinking aloud is claimed to hinder the operation of special, non-reportable processes. Whilst verbal overshadowing was not evident in final solution rates, there was nevertheless support for verbal overshadowing up to and beyond the mid-point of the available problem solving time. Overall our data support a special-process theory of insight, whilst also pointing to the role of moderator variables (e.g., available time for solution) in determining the presence or absence of effects predicted by the special-process account.
\end{abstract}

\title{
Keywords
}

Insight problem solving; distraction; articulatory suppression; irrelevant speech; thinking aloud; verbal overshadowing 


\section{When Distraction Helps: Evidence that Concurrent Articulation and Irrelevant Speech Can Facilitate Insight Problem Solving}

The phenomenon of "insight" in problem solving and creative thinking arises when an individual who is stuck on a task and is unable to make any headway suddenly finds that they are able to generate a successful solution (e.g., Kaplan \& Simon, 1990). This phenomenon appears to have three characteristic features. First, having spent time attempting the problem, individuals reach a point of impasse from which they are unable to move forward to achieve a solution, perhaps because of the continued application of mistaken assumptions about the nature of the problem (e.g., Dominowski \& Dallob, 1995). Second, the insight into the solution emerges suddenly - giving rise to a so-called "Aha!" experience - rather than arising as a result of incremental processes that bring people progressively closer to the solution (e.g., Metcalfe \& Weibe, 1987; Smith \& Kounios, 1996). Third, problem solvers find it difficult to describe the processes that helped them overcome the impasse, perhaps because of the rapidity with which the solution came to mind (e.g., Maier, 1931) or alternatively because the underlying solution processes are simply non-reportable in nature (e.g., Knoblich, Ohlsson, \& Raney, 2001).

Some researchers have questioned whether the aforementioned characteristic features of insight problem solving are strictly necessary. For example, Fleck and Weisberg (2004) observed that solutions frequently arose on a classic insight problem in the absence of a period of impasse, whilst Ohlsson (1992) has disputed the notion that complete solutions suddenly become available to consciousness. Notwithstanding these definitional concerns, it is readily apparent that these supposed features of insight problems have informed the dominant account of insight problem solving in the literature - the so-called "specialprocess" theory (e.g., Bowden, Jung-Beeman, Fleck, \& Kounios, 2005). This theory embraces the idea that insight and non-insight problems invoke very different processing 
mechanisms. In the case of insight problems, the core claim of the special-process theory is that insight is driven by the operation of non-reportable processes that function at an implicit and non-conscious level in order to change the current, unsuccessful representation of a problem into a new, "re-structured" representation that can lead to a solution (e.g., see Bowden et al., 2005).

The non-reportable, implicit processes that are emphasised within the special-process theory of insight include, in particular, spreading activation in semantic memory (e.g., Ohlsson, 2011) and the switching from fine-grained semantic processing in the lefthemisphere to more coarse-grained semantic processing in the right hemisphere (e.g., JungBeeman et al., 2004). By highlighting processes such as these, which are linked to the diffuse semantic activation of alternative concepts and distant associations, the special-process theory builds upon Ohlsson's (1992) pioneering "representational-change" account of insight, which suggests three key ways in which a problem's representation may be changed through implicit processes so as to enable solution success, that is: (1) elaboration, where new information about the problem is added to the original representation; (2) re-encoding, where the problem is reinterpreted in a different, more successful manner; and (3) constraint relaxation, where self-imposed constraints are relaxed, thereby enabling progress to be made.

The experiment that we report in the present paper aimed to provide new evidence to support a special-process view of insight by demonstrating that it is possible to facilitate the operation of such special processes that occur at an unconscious (and therefore nonreportable) level. Our experiment was predicated on the assumption that if the opportunity to engage in conscious, speech-based processing of insight problems is substantially reduced or disrupted then this should enable more effective special processing to take place at a nonconscious level that can more readily gain strength and emerge into consciousness. To explain the basis of this assumption we appeal to the key proposals of special-process 
theorists, who argue that the initial, conscious and reportable processing of insight problems will tend to focus on strongly activated information that is, in fact, unrelated to a solution, whereas weakly activated information that is critical for solution success may be unable to enter consciousness because it is blocked or overshadowed by the stronger and reportable (but misdirected) information (e.g., see Bowden et al., 2005; Kershaw \& Ohlsson, 2004; Siegler, 2000). What this means, therefore, is that enhancing the opportunity for problem solvers to take full advantage of weaker solution activation under conditions of reduced speech-based processing should serve to increase the likelihood of solution success and reduce problem solving latencies. Producing such evidence for problem-solving benefits arising from reduced speech-based processing would clearly provide good support for the role of special, non-reportable processes in insight problem solving.

As noted, a key aspect of the benefits that we predict for insight solutions from reduced speech-based processing relates to the possibility of undermining so-called "verbal overshadowing" effects in insight problem solving. Traditionally, such verbal overshadowing has been claimed to arise from the experimenter enforcing a "think aloud" requirement on the participant during problem solving (Schooler, Ohlsson, \& Brooks, 1993; see also Schooler \& Melcher, 1995), with such thinking aloud being claimed to direct attention toward aspects of the problem that can easily be verbalized but which are inappropriate for finding a solution. As we have suggested above, we believe that an element of subvocal verbal processing is also likely to be a normal aspect of "silent" problem solving, such that a degree of verbal overshadowing might well arise in insight tasks even without the direct imposition of an explicit think aloud requirement. Notwithstanding this assumption regarding the disruptive effects of such subvocal verbal processing on insight problem solving, our experiment also afforded an opportunity to set up a condition to test for heightened levels of verbal overshadowing arising from the implementation of an explicit think aloud instruction. 
Given our core interest in verbal overshadowing phenomena in insight problem solving it is important to note that the standard verbal overshadowing effect that has been claimed to arise from a think aloud requirement has only been replicated inconsistently in the context of insight tasks. Although good replicability was initially reported that extended to domains such as visual problem solving (DeShon, Chan, \& Weissbein, 1995) and analogical reasoning (Sieck, Quinn, \& Schooler, 1999), it is now apparent that there had been a declining profile of replications over time (for examples of recent, failed replication with insight problems see Ball \& Stevens, 2009; Chein \& Weisberg, 2014; Chein, Weisberg, Streeter, \& Kwok, 2010; Gilhooly, Fioratou, \& Henretty, 2010; Fleck \& Weisberg, 2004, 2013). This diminishing evidence for verbal overshadowing appears to be a case of a "decline effect", whereby an initially striking scientific phenomenon shows a diminishing effect size over time (see Lehrer, 2010). Because of space considerations we will not engage in further discussion of such decline effects and their potential causes, which are only partly understood. Suffice it to say that questions remain as to whether verbal overshadowing arising from think aloud instructions is a robust phenomenon and that the present study permitted a further opportunity to test for the effect in the specific case of insight problem solving.

\section{Evidence for the Special-Process Theory of Insight}

In addition to the evidence for verbal overshadowing of insight there are several other sources of evidence that lend credence to the special-process theory of insight. For example, insight problems embodying constraints that are difficult to relax because of prior domain knowledge and assumptions are found to be particularly hard to solve, as predicted by the representational-change theory (e.g., Knoblich, Ohlsson, Haider, \& Rhenius, 1999; Knoblich, et al., 2001; see also Murray \& Byrne, 2013). Other studies have shown that the thought processes that arise just before an insight solution are non-conscious in nature. For example, 
Bowden and Jung-Beeman (1998) presented participants with still-to-be-solved problems requiring a single-word solution as well as either the solution word or an unrelated word. Bowden and Jung-Beeman found that participants read the solution word faster than the unrelated word, suggesting that unconsciously they already knew the solution before becoming consciously aware of it (see also Siegler, 2000).

Further influential support for the special-process theory of insight has been derived from neuroimaging studies, which have clarified how insight problem solving differs from other types of problem solving in terms of underlying neurological activity. In this respect Bowden and Jung-Beeman (1998) suggested that to solve insight problems the individual often has to retrieve associations that are "indistinctly related" to features of the presented task. As we noted above, these authors have proposed that this type of diffuse associative processing occurs in the right hemisphere, which is capable of coarse semantic coding such as the weak activation of alternative problem associations. In contrast, the left hemisphere is specialized for fine semantic coding, which focuses activation on a single problem interpretation. Prior to insight, fine semantic coding can cause misdirection by concentrating attention on irrelevant problem components such that the solver in unable to see past an initial interpretation to find a solution. Bowden and Jung-Beeman (1998) tested these assumptions by presenting to each visual field either solution words or unrelated words for unsolved, verbally-based insight problems. Results demonstrated a left visual field (righthemisphere) advantage for these solution-words.

Other neuroimaging studies support the idea that insight arises from activation localized in the right anterior superior temporal gyrus (Bowden et al., 2005). In addition, EEG recordings have revealed a sudden burst of high frequency (gamma band) neural activity in this right hemisphere region immediately preceding insight. It seems that solvers 
change the focus of their efforts just before insight, thereby allowing previously nonreportable, right-hemisphere solution-information to emerge into consciousness.

\section{The Business-as-Usual Theory of Insight}

Notwithstanding the diversity of experimental and neuroscience evidence supporting the special-process theory of insight, a number of theorists argue for a contrary position, which has been referred to as the "business-as-usual" approach (e.g., see Bowden et al., 2005). Theorists supporting this position claim that insight and non-insight problems, whilst differing in terms of key features such as their tendency to induce an impasse state, are still tackled and solved using essentially equivalent cognitive mechanisms (Weisberg, 2006). These business-as-usual mechanisms include strategies such as means-ends analysis and hillclimbing (Newell \& Simon, 1972), which lead to the step-by-step attainment of solutions.

A good example of a business-as-usual approach is the "progress-monitoring theory" developed by MacGregor, Ormerod, and Chronicle (2001; see also Chronicle, Ormerod, \& MacGregor, 2004; Ormerod, MacGregor, Chronicle, Dewald, \& Chu, 2013), which proposes that solvers aim to minimize the difference between their current "problem state" and the "goal state" regardless of whether a problem is an insight or non-insight task. Accordingly, insight is simply the realisation that a goal state is not achievable with the remaining available moves such that new moves must be devised to attain the solution.

\section{Aims of the Experiment}

The evidence supporting the special-process account of insight seems compelling, although we also note Bowden et al.'s (2005) cautionary suggestion that both special-process and business-as-usual theories may be correct inasmuch as there may be multiple ways in which insight can arise (cf. Fleck \& Weisberg, 2013; see also Weisberg, this issue, for an integrated view of insight that recognises the role of both special processes and business-asusual mechanisms). The experiment that we report here aimed to inform the debate 
concerning theories of insight by establishing conditions that would enable competing predictions to be tested. Our experimental set-up involved four between-participants conditions: silent working, articulatory suppression, irrelevant speech and thinking aloud. We explain these conditions and the associated predictions in more detail below. All participants tackled three visuo-spatial insight problems (Appendix A), each possessing single, unambiguous solutions, the attainment of which involve information restructuring.

Our first condition was a control condition in which participants worked silently on the target problems. This provided a baseline measure of solution success. Our second condition required participants to engage in articulatory suppression (repeatedly speaking aloud a stereotypic number sequence) whilst attempting each insight problem. This articulatory suppression requirement aimed to suppress inner speech within the articulatory control process that is integral to the phonological loop component of working memory (Baddeley, 2007). This articulatory control process is believed to be heavily involved in speech planning, subvocal rehearsal and the conversion of visually presented material into a phonological code (e.g., Repovš \& Baddeley, 2006) ${ }^{1}$. It was therefore assumed that articulatory suppression would inhibit the use of internal, speech-based processing during problem solving, which, in line with the special-process account, should lead to enhanced insight by enabling more effective special processes to take place at a non-conscious level such that weakly activated information can strengthen and shine through into consciousness. In contrast, the business-as-usual account would predict worse insight performance arising under articulatory suppression given its disruptive influence on conscious, strategic analysis and planning, which are presumably underpinned by speech-based processing. At the very least the distraction arising from articulatory suppression would be expected to disrupt processes such as means-ends analysis and hill climbing. 
Our third condition involved presenting participants with irrelevant speech in the form of a looped sequence of ascending digits (from 1 to 7) spoken repeatedly in an even-pitched (i.e., monotone) voice. Irrelevant speech is highly distracting to task-based processing, with evidence increasingly supporting a "duplex-mechanism" account to explain two distinct auditory distraction effects (e.g., see Hughes, Hurlstone, Marsh, Vachon, \& Jones, 2013). One effect, referred to as "interference-by-process" (Hughes et al., 2013), arises from a conflict between the obligatory processing of irrelevant speech and the speech-based processing deployed to perform the target task (e.g., Hughes, Tremblay, \& Jones, 2005). The other effect, referred to as "attentional capture" (Hughes et al., 2013), concerns the disruption of processing arising simply because irrelevant speech draws attention away from the target task. In relation to our present concerns with the impact of auditory distraction on insight problem solving we were especially interested in the interference-by-process effect arising from irrelevant speech, since such interference would be likely to reduce a participant's capacity to engage in speech-based processing whilst attempting the given problems. As with articulatory suppression, we therefore predicted that irrelevant speech would - according to a special-process account - serve to enhance insight problem solving by reducing opportunities for internal, speech-based processing of the insight tasks. In contrast, the business-as-usual account would again predict worse performance on the insight problems in the face of irrelevant speech because of the distraction caused to conscious strategic planning and analysis.

Our final, "thinking aloud" condition required participants to verbalize all thoughts passing through their minds whilst attempting to solve the insight problems. Such thinking aloud encourages explicit and focused verbal processing of the presented problems (Schooler et al., 1993) and therefore stands in marked contrast to the situations involving articulatory suppression or irrelevant speech, where verbal processing of problems is largely unavailable 
to participants. As already explained, from a special-process perspective the thinking aloud requirement is predicted to overshadow the non-conscious processes that underpin insight problem solving. In contrast, the business-as-usual account would predict no real impact on insight performance from participants having to verbalize their thoughts since people are simply reporting information that would normally be heeded during the application of strategies such as means-ends analysis (cf. Ericsson \& Simon, 1993; see also Gilhooly et al., 2010). A business-as-usual account might even predict that thinking aloud could have a beneficial effect on solution latencies and frequencies since there is evidence that verbalization can help solvers maintain a focus on task goals and strategies (Chi, 2000).

\section{Method}

\section{Participants}

The study involved 96 participants (57 female) who were students or administrative staff at Lancaster University and the University of Central Lancashire. The age range of participants was 18 to 62 years (mean age: 26.4 years). Participants received either a small fee or course credit for undertaking the study. None had received previous tuition relating to the psychology of problem solving.

Design

The experiment involved a between-participants design with four conditions: (1) articulatory suppression (attempting each presented insight problem whilst concurrently articulating a stereotypic number sequence); (2) irrelevant speech (attempting each problem whilst ignoring a stereotypic number sequence); (3) thinking aloud (attempting each problem whilst concurrently speaking out load all thoughts); and (4) silent working (attempting each problem quietly, which acted as a control condition). Participants had a maximum of 7.5 minutes to tackle each of the insight problems and the time taken to solve each problem correctly was recorded using a stop-watch. 


\section{Materials}

Insight problems. Participants were asked to tackle three insight problems presented in pictorial form, which each required visuo-spatial manipulations for correct solution attainment (Appendix A). The "pigs in a pen" problem (e.g., Schooler et al., 1993) involves a starting state where nine pigs (depicted as icons of pigs' faces) are enclosed within a square pen. Participants are asked to construct two more square pens so as to ensure that each of the pigs ends up in a pen of its own. The solution to this problem entails the insight that one of the new square pens needs to be drawn in a rotated form as a diamond shape (Appendix A). The problem is known to be challenging, with Schooler et al. (1993) indicating that mean solution rates were close to $50 \%$, although precise data were not specified.

The "triangle" problem (e.g., de Bono, 1969; Schooler et al., 1993) involves the presentation of 10 identically-sized circles that form the shape of a triangle pointing towards the top of the page. Participants are asked to make the triangle shape point downwards by moving only three of the circles. The solution to this problem (Appendix A) is known to be difficult, with Schooler et al. (1993) suggesting that solution rates were again close to $50 \%$.

The "deer" problem (origin unknown) involves presenting a stick-figure of a deer that is depicted using five lines of equal length. Participants are asked to make the deer face in a different direction by moving just one of the lines. The solution to this problem entails moving the deer's back leg to the end of the front leg (Appendix A), with this insight, again, being challenging to achieve.

Each problem was presented on a separate piece of A4 paper with the statement of the problem at the top of the page. Ample blank space was available below each problem to enable participants to pursue rough workings whilst attempting to derive a solution. A final solution could be presented to the experimenter either verbally or visually. 
Experimental conditions and associated instructions: In addition to the silent working (control) condition there were three experimental conditions: articulatory suppression, irrelevant speech and thinking aloud. The instructions in the articulatory suppression condition asked participants to count aloud continuously from 1 to 7 whilst attempting to solve the insight problems. In the irrelevant speech condition participants were instructed that whilst attempting to solve the insight problems they would be presented with irrelevant speech over a set of headphones. They were told that the speech sounds were irrelevant to the problem solving task and that they should ignore the speech as best as they could. In the thinking-aloud condition participants were asked to verbalize all of their thoughts out loud whilst attempting to solve the insight problems.

\section{Procedure}

Participants were tested one at a time and were given a pen and a booklet containing three insight problems (one per page) plus a cover sheet that provided general instructions regarding the number of problems that they would tackle, how long they had per problem and how to register responses. The cover sheet also presented condition-specific instructions, as explained below. The order of presentation of the three insight problems was counterbalanced using a balanced Latin square design, which resulted in six different problem orderings that were repeated across the 24 participants in each condition.

At the commencement of the experiment participants were asked to complete a consent form and to read the general instructions. Any questions were answered by the experimenter at this point before participants were then asked to turn to the first problem and to read it aloud. The timing of the solution attempt for each problem was made using a digital stop-watch, with timing commencing as soon as the participant started reading the problem. Participants in all four conditions were asked to begin working on each problem immediately after reading it. Participants in the articulatory suppression condition were required to 
rehearse overtly the number sequence " $1,2,3,4,5,6,7$ ” as soon as they had read each problem and were informed that each digit in the sequence should take about a half a second or less to say. Participants in the irrelevant speech condition were repeatedly presented with the pre-recorded number sequence " $1,2,3,4,5,6,7$ " as soon as they had read each problem, with the numbers spoken in a monotone male voice at a rate of two digits per second. Participants in the think-aloud condition were required to verbalize all of their thoughts whilst tackling each problem. They were informed that they should find this quite easy to do, since it simply involved saying out loud whatever was passing through their mind when trying to solve each problem. They were also told that they would be prompted to keep thinking aloud should they fall silent for more than a few seconds. In both the articulatory suppression and think-aloud conditions participants' verbalizations were recorded using an MP3 player in order to reinforce the importance of their spoken activity. These recorded data were not retained for further analysis.

The stop-watch was paused when the participant notified the experimenter that they had solved the problem. This time was recorded as the solution time if the participant was able to demonstrate the correct solution to the experimenter - typically by referring to their sketched depiction of the solution. If the proffered solution was incorrect then the stop-watch was re-started and the participant was asked to continue tackling the problem if there was still time remaining. If the participant reached the maximum permissible time of 7.5 minutes without having solved the problem then this was noted. The same procedure was adopted for all three problems and the experimenter told participants when they could move onto the next problem. After completing the experiment a full experimental debrief was provided.

\section{Results and Discussion}

The proportion of problems solved over time and across conditions was examined statistically by means of a survival analysis in SPSS using the non-parametric Kaplan-Meier 
method given that all assumptions of this method were met (Kaplan \& Meier, 1958). This analysis technique is particularly well suited for assessing the present dataset since it enables comparisons to be made across conditions in terms of the cumulative proportion of problems solved within a specified time-frame, whilst at the same time being sensitive to the relatively high rate of unsuccessful solutions (i.e., censored cases) that typically arises in studies of insight problem solving. We have recently used survival analysis to good effect in our previous research on insight problem solving, where we examined how solution likelihood is enhanced when participants' observe eye-movement cues that "embody" solution principles associated with a correct solution (Litchfield \& Ball, 2011). To compare survival functions statistically in the present study we used the commonly applied Mantel-Cox log-rank test (e.g., see Collett, 2003), which is a goodness-of-fit test (in this case a chi-square test) that examines the equivalence of the cumulative survival distributions across conditions. If an overall significant difference in survival distributions is identified across multiple conditions then this can be unpacked by means of pairwise comparisons that again employ the log-rank test.

\section{Proportion of Problems Solved across Conditions over Time}

In our first analysis we examined the data collapsed across participants within each condition, effectively treating each condition as containing a set of 72 items (i.e., 3 problems x 24 participants), with each item either being solved at a particular point in time or else remaining unsolved after 7.5 minutes (i.e., 450 seconds). Figure 1 therefore depicts the proportion of problems solved in each condition at various points in time, with the most vertical data-point on the curve at any particular time-point reflecting the fact that an insightful solution had been registered at that time by a participant in that condition. 
An analysis of the data shown in Figure 1 revealed a significant difference in the proportion of problems solved across conditions, $\chi^{2}(3, \mathrm{~N}=288)=17.71, p<.001$. Pairwise comparisons indicated that participants in the articulatory suppression condition were more likely to solve the problems than participants in either the thinking aloud condition, $\chi^{2}(1, \mathrm{~N}=$ $96)=12.91, p<.001$, or the silent working (control) condition, $\chi^{2}(1, \mathrm{~N}=96)=6.65, p=.01$. This finding supports our prediction that the distraction arising from articulatory suppression would have a beneficial effect on insight by virtue of its disruptive effect on inner speech in terms of reducing opportunities for the verbally-based processing of problems. Similarly and again as predicted - the participants in the irrelevant speech condition were more likely to solve the problems than participants in either the thinking aloud condition, $\chi^{2}(1, \mathrm{~N}=96)=$ $10.66, p<.001$, or the silent working condition, $\chi^{2}(1, \mathrm{~N}=96)=4.53, p=.03$. This latter finding again attests to the beneficial role that distraction can have on insight problem solving when such distraction influences the capacity for speech-based processing.

Our follow-up analyses also revealed that there was no difference between the articulatory suppression and the irrelevant speech conditions, $\chi^{2}(1, \mathrm{~N}=96)=.42, p=.52$. This finding gives further grounds for viewing the facilitatory effects of distraction in these two conditions as having a common basis that is linked to the disruption of internal, speechbased processing. One further pairwise comparison was conducted to examine the critical difference between the thinking aloud and silent working conditions, with a view to assessing the presence of verbal overshadowing of insight solutions arising from the enforced verbalization requirement in the thinking aloud condition. This comparison was not found to be significant, $\chi^{2}(1, \mathrm{~N}=96)=.35, p=.56$, although visual inspection of the data (Figure 1 ) suggests that thinking aloud inhibited early solution success during the initial period of problem solving associated with each insight task. Indeed, during the first half of problem solving participants seemed to be fairly impoverished in their capacity to solve the insight 
problems when thinking aloud, whilst the second half of the available time revealed a period of recovery in this condition, with participants ending up having very similar solution rates to those who had worked silently.

Proportion of Problems Solved across Conditions up to the Mid-Point of the Available Time

These latter observations were corroborated in a subsequent analysis (Figure 2), where we analyzed problem solving success across conditions up the mid-point of the available time per task (i.e., up to the first 225 seconds of the total 450 seconds available problem solving time). This new analysis revealed a significant difference across conditions, $\chi^{2}(3, \mathrm{~N}=288)=33.79, p<.001$, and pairwise comparisons confirmed that participants in the thinking-aloud condition were significantly less likely to solve the problems than participants in the other conditions, including the silent working condition (all $p s<.002$ ). This finding is important in terms of the support that it lends to Schooler et al.'s (e.g., 1993) verbal overshadowing hypothesis, since it does indeed appear that thinking aloud is highly disruptive to insight problem solving, although in the present study it appears that such disruption has a temporal dimension, being particularly evident during the initial phase of problem solving but then dissipating later on.

***Please insert Figure 2 about here***

Restricting the analysis to the mid-point of the available time not only clarified that the thinking aloud condition led to significantly worse problem solving performance than the silent working control condition (i.e., a verbal overshadowing effect), but also revealed apparent differences between the positive impact of articulatory suppression and irrelevant speech when pitted against silent working. That is, participants in the articulatory suppression condition were significantly more likely to solve problems than those in the silent working condition, $\chi^{2}(1, \mathrm{~N}=96)=7.49, p=.006$, whereas the difference in solution rates between the irrelevant speech the silent working conditions only approached significance, $\chi^{2}(1, \mathrm{~N}=$ 
$96)=3.17, p=.075$. The strength of the latter difference between irrelevant speech versus silent working may, however, have been diluted because of the presence of data for the deer problem in the analysis, which was a problem that gave rise to poor solution rates in all conditions $^{2}$. Indeed, removing the deer problem from the dataset revealed that the articulatory suppression and irrelevant speech conditions produced equivalent enhancement effects relative to silent working, that is, articulatory suppression versus silent working: $\chi^{2}(1, N=$ $24)=6.38, p=.01$; irrelevant speech versus silent working: $\chi^{2}(1, \mathrm{~N}=24)=4.75, p=.03$.

\section{General Discussion}

The present study aimed to inform the debate as to whether insight problem solving relies on implicit, non-conscious and indescribable processes (the special-process view; e.g., Bowden et al., 2005) as opposed to explicit, conscious and describable processes (the business-as-usual view; e.g., MacGregor et al., 2001). Our experiment was designed primarily to test the novel prediction that reducing opportunities for speech-based processing during insight problem solving should facilitate successful solution attainment. This facilitation effect was expected to arise since distracting internalized speech-based thought processes by means of techniques such as articulatory suppression and the presentation of irrelevant speech should permit the occurrence of more effective non-conscious and nonreportable processes, thereby enabling weakly activated solution concepts to strengthen and spill over into conscious awareness. This is precisely the type of "special" processing that is claimed to underpin the release from impasse to an insightful solution via problem restructuring (e.g., Ohlsson, 1992, 2011; Bowden et al., 2005).

A secondary objective of our experiment was to test directly for the presence of verbal overshadowing in insight problem solving (Schooler et al., 1993) by including a condition in which participants were required to think aloud whilst tackling problems. A verbal overshadowing effect is predicted by the special-process theory of insight, since thinking 
aloud is believed to bias processing toward a verbalizable, conscious form that interferes with the special, unreportable processes required for insight (Schooler et al., 1993; Schooler \& Melcher, 1995).

The target insight tasks that participants were asked to solve were three visuo-spatial problems that are believed to give rise to the classic insight sequence of initial, failed solution attempts, followed by impasse, which in turn is followed by the sudden emergence of an insightful solution. As part of our between-participants experimental design we included a silent working condition to provide a baseline measure of performance against which we could compare our three experimental conditions: articulatory suppression, irrelevant speech and thinking aloud.

Our findings appear to be broadly supportive of the special-process theory of insight and, by implication, seem incompatible with the business-as-usual account. Of particular note is the novel evidence that the two conditions associated with the distraction of speech-based processing are also the conditions associated with a significantly increased likelihood of insightful solutions being attained relative to either the thinking aloud or silent working conditions. In other words, inhibiting opportunities for any speech-based processing of the presented problems facilitates the emergence of insightful solutions, presumably by allowing non-conscious and non-reportable processes to operate successfully in re-structuring the problem. Furthermore the similar magnitude of the benefits for insight problem solving arising from articulatory suppression and irrelevant speech seems to implicate a common factor underpinning the facilitation effect. This factor is presumably linked to the way in which articulatory suppression and irrelevant speech both interfere with processes such as speech planning and subvocal rehearsal (e.g., Hughes et al., 2005, 2013; Repovš \& Baddeley, 2006). 
In relation to the impact of thinking aloud on insight, our comparison between the thinking aloud condition and the silent working condition revealed no negative effect of thinking aloud on participants' final likelihood of achieving an insightful solution. By the end of the available time the percentage of problems solved was $56 \%$ in the thinking aloud condition, which was, in fact, slightly better than the $53 \%$ observed in the silent working condition. At first sight such similar final solution probabilities seems to run counter to the presence of any verbal overshadowing effect arising from thinking aloud. Interestingly, however, this analysis of final solution rates obscures a more interesting pattern that is discernable in the data when looking at solution likelihood over the full time-course of problem solving. What is evident from a temporal analysis for the thinking aloud condition is the presence of severely diminished initial performance relative to the silent working condition that extends well past the mid-point of the available problem solving period, with a late recovery in performance occurring rapidly toward the end of the available time. This temporal pattern (i.e., early onset of a striking verbal overshadowing effect arising from thinking aloud versus late release from this harmful effect) was corroborated in a statistical comparison between the thinking aloud and silent working conditions that was restricted to the time period stretching from the beginning to the mid-point of problem solving activity.

The evidence that the verbal overshadowing effect may have a temporal dimension is important given current controversies surrounding the reality of the verbal overshadowing phenomenon in the context of insight problem solving (e.g., Ball \& Stevens, 2009; Chein \& Weisberg, 2014; Chein et al., 2010; Fleck \& Weisberg, 2004, 2013). It could be, for example, that studies presenting positive evidence of the verbal overshadowing of insight are ones that involve a reduced period of available time for problem solving (i.e., somewhat less than the 7.5 minutes permitted in the present study), whereas studies that fail to reveal a verbal 
overshadowing effect are ones that examine final solution rates at the end of a lengthy time period akin to that available in our experiment.

In this latter respect it is noteworthy that Schooler et al.'s (1993) Experiment 3, which revealed a verbal overshadowing effect and is their reported experiment that is aligned most closely to our one in terms of methodology, involved a maximum of 6 minutes of problem solving time per item, which is 1.5 minutes less per item than in our study. A final solution time of 6 minutes (i.e., 360 seconds), would fall at a time-point in our dataset (Figure 1) where there is a sizeable difference in solution likelihood for the thinking aloud condition versus the silent working condition (i.e., where verbal overshadowing of insight appears to be in evidence). In contrast, Fleck and Weisberg's (2013) recent replication failure of a verbal overshadowing effect in a study that included up to eight insight tasks per participant involved a maximum time limit of 10 minutes per item (also see Fleck \& Weisberg, 2004, for an earlier replication failure of verbal overshadowing with a single insight task that also had a 10 minute problem solving window). Clearly 10 minutes of available problem solving time is well past the 7.5 minutes that we allowed in our study and would thereby provide ample opportunities for a late release from overshadowing, similar to what we observed.

The idea that available time might be a critical factor for the emergence of verbal overshadowing in insight problem solving seems worthy of further investigation. Indeed, this factor might help to account for the decline effect seen in published studies of the verbal overshadowing phenomenon, especially if there has been a tendency for more recent studies to implement longer solution durations per problem. As is often the case with cognitive phenomena that seem robust to begin with but which show subsequent replication failures, there are typically key moderator variables at work that impose boundary conditions on when the phenomenon is likely to be observed (e.g., see Thompson et al., 2013, for a recently suggested cognitive ability factor that may moderate the beneficial effects of processing 
"disfluency" on successful reasoning performance). In the case of failures to replicate verbal overshadowing of insight then available problem solving time might be one important moderator, whilst other moderator variables may also be implicated, including whether target problems are primarily visuo-spatial in nature, as in the present study, or verbally-based, as in numerous recent studies where no verbal overshadowing has been observed (e.g., Ball \& Stevens, 2009; Chein \& Weisberg, 2014; Chein et al., 2010; see also Gilhooly et al., 2010, for a comparison of verbal overshadowing with verbal vs. visuo-spatial insight tasks).

In relation to the replicability of the verbal overshadowing effects in insight problem solving we finally note that even when working silently it is still possible for problem solvers to engage in internalized (subvocal) verbal processing that could be disruptive to the special, non-reportable processes that may underpin insightful solutions. Indeed our experimental predictions concerning the beneficial effects of articulatory suppression and irrelevant speech on insight were underpinned by an assumption about the likely involvement of such subvocal verbal processing in thwarting insight. With these points in mind we note that the effect size associated with the verbal overshadowing phenomenon arising from think aloud instructions could well vary substantially from one experiment to another, dependent on the make-up of samples in terms of participants' inherent tendencies to engage in internalized speech-based processing. In other words, variability in processing styles (e.g., verbal vs. visuo-spatial) that have been claimed to arise in thinking and reasoning (e.g., Ford, 1994), could well serve either to enhance or diminish the emergence of verbal overshadowing effects in insight problem solving. This issue represents another worthwhile avenue for future research that could further inform concerns over the reliability of the verbal overshadowing effect and the evidence that verbal overshadowing provides for the special-process theory of insight problem solving. 
To conclude, we believe that our study has provided original and unique evidence to support a special-process view of insight problem solving. By using experimental techniques that inhibit people's ability to apply speech-based processes to tackle insight problems we have shown that special, non-reportable processes are able to shine through and dominate responding, thereby promoting the attainment of insightful solutions. This seems to be an interesting and perhaps counterintuitive case of distraction actually serving to facilitate problem solving success, although we maintain that this is a phenomenon that makes good sense when viewed from the perspective of the special-process theory of insight. 
Distraction Facilitates Insight Problem Solving

\section{References}

Baddeley, A. D. (2007). Working Memory, Thought and Action. Oxford: Oxford University Press.

Ball, L. J., \& Stevens, A. (2009). Evidence for a verbally-based analytic component to insight problem solving. In N. Taatgen \& H. van Rijn (Eds.), Proceedings of the 31st Annual Conference of the Cognitive Science Society. Austin, TX: Cognitive Science Society.

Bowden, E. M., \& Jung-Beeman, M. (1998). Getting the right idea: Semantic activation in the right hemisphere may help solve insight problems. Psychological Science, 6, 435-440.

Bowden, E. M., Jung-Beeman, M., Fleck, J., \& Kounios, J. (2005). New approaches to demystifying insight. Trends in Cognitive Sciences, 9, 322-328.

Chein, J. M., \& Weisberg, R. W. (2014). Working memory and insight in verbal problems: Analysis of compound remote associates. Memory \& Cognition, 42, 67-83.

Chein, J. M., Weisberg, R. W., Streeter, N. L., \& Kwok, S. (2010). Working memory and insight in the nine-dot problem. Memory \& Cognition, 38, 883-892.

Chi, M. T. H. (2000). Self-explaining expository texts: The dual processes of generating inferences and repairing mental models. In R. Glaser (Ed.), Advances in Instructional Psychology (pp. 161-238). Hillsdale, NJ: Lawrence Erlbaum Associates.

Chronicle, E. P., Ormerod, T. C., \& MacGregor, J. N. (2004). What makes an insight problem? The role of heuristics, goal conception and solution recoding in knowledge-lean problems. Journal of Experimental Psychology. Learning, Memory \& Cognition, 30, 14-27.

Collett, D. (2003). Modelling Survival Data in Medical Research. London, UK: Chapman Hall.

De Bono, E. (1969). The Mechanism of Mind. New York: Penguin. 
DeShon, R. P., Chan, D., \& Weissbein, D. A. (1995). Verbal overshadowing effects on Raven's Advanced Progressive Matrices: Evidence for multidimensional performance determinants. Intelligence 21, 135-155.

Dominowski, R., \& Dallob, P. (1995). Insight and problem solving. In R. J. Sternberg \& Davidson, J. (Eds.), The Nature of Insight (pp. 33-62). Cambridge, MA: MIT Press.

Ericsson, K. A., \& Simon, H.A. (1993). Protocol Analysis: Verbal Reports as Data (Revised Edition). Cambridge, MA: MIT Press.

Fleck, J. I., \& Weisberg, R. W. (2004). The use of verbal protocols as data: An analysis of insight in the Candle Problem. Memory \& Cognition, 32, 990-1006.

Fleck, J. S., \& Weisberg, R. W. (2013). Insight versus analysis: Evidence for diverse methods in problem solving. Journal of Cognitive Psychology, 25, 436-463.

Ford, M. (1994). Two modes of mental representation and problem solution in syllogistic reasoning. Cognition, 54, 1-71.

Gilhooly, K. J., Fioratou, E., \& Henretty, N. (2010). Verbalization and problem solving: Insight and spatial factors. British Journal of Psychology, 101, 81-93.

Hughes, R. W., Hurlstone, M. J., Marsh, J. E., Vachon, F., \& Jones, D. M. (2013). Cognitive control of auditory distraction: Impact of task difficulty, foreknowledge and working memory capacity supports duplex-mechanism account. Journal of Experimental Psychology: Human Perception \& Performance, 39, 539-553.

Hughes, R. W., Tremblay, S., \& Jones, D. M. (2005). Disruption by speech of serial short-term memory: The role of changing-state vowels. Psychonomic Bulletin \& Review, 12, 886-890.

Jones, D. M., Hughes, R. W., \& Macken, W. J. (2006). Perceptual organization masquerading as phonological storage: Further support for a perceptual-gestural view of short-term memory. Journal of Memory \& Language, 54, 265-281. 
Jones, D. M., Hughes, R. W., \& Macken, W. J. (2007). The phonological store abandoned. Quarterly Journal of Experimental Psychology, 60, 497-504.

Jones, D. M., Macken, W. J., \& Nicholls, A. P. (2004). The phonological store of working memory: Is it phonological, and is it a store? Journal of Experimental Psychology: Learning, Memory, and Cognition, 30, 656-674.

Jung-Beeman, M., Bowden, E. M., Haberman, J., Frymiare, J. L., Arambel-Liu, S., Greenblatt, R.,..., Kounios, J. (2004). Neural activity when people solve verbal problems with insight. PLoS Biology, 2, E97, 500-510.

Kaplan, C., \& Simon, H. A. (1990). In search of insight. Cognitive Psychology, 22, $374-419$.

Kaplan, E. L., \& Meier, P. (1958). Nonparametric estimation from incomplete observations. Journal of the American Statistical Association, 53, 457-485.

Kershaw, T. C., \& Ohlsson, S. (2004). Multiple causes of difficulty in insight: The case of the nine-dot problem. Journal of Experimental Psychology: Learning, Memory, and Cognition, 30, 3-13.

Knoblich, G., Ohlsson, S., Haider, H., \& Rhenius, D. (1999). Constraint relaxation and chunk decomposition in insight problem solving. Journal of Experimental Psychology: Learning, Memory \& Cognition, 25, 1534-1555.

Knoblich, G., Ohlsson, S., \& Raney, G. E. (2001). An eye movement study of insight problem solving. Memory \& Cognition, 29, 1000-1009.

Lehrer, J. (2010). The truth wears off: Is there something wrong with the scientific method. The New Yorker, December 13 2010, 52-57.

Litchfield, D., \& Ball, L. J. (2011). Using another's gaze as an explicit aid to insight problem solving. Quarterly Journal of Experimental Psychology, 64, 649-656. 
MacGregor, J. N., Ormerod, T. C., \& Chronicle, E. P. (2001). Information-processing and insight: A process model of performance on the nine-dot and related problems. Journal of Experimental Psychology: Learning, Memory \& Cognition, 27, 176-201.

Maier, N. R. F. (1931). Reasoning in humans: II. The solution of a problem and its appearance in consciousness. Journal of Comparative Psychology, 12, 181-194.

Metcalfe, J., \& Weibe, D. (1987). Intuition in insight and non-insight problem solving. Memory \& Cognition, 15, 238-246.

Murray, M. A., \& Byrne, R. M. J. (2013). Cognitive change in insight problem solving: Initial model errors and counterexamples. Journal of Cognitive Psychology, 25, 210 219.

Newell, A., \& Simon, H. A. (1972). Human Problem Solving. Englewood Cliffs, NJ: Prentice-Hall.

Ohlsson, S. (1992). Information processing explanations of insight and related phenomena. In M. T. Keane \& K. J. Gilhooly (Eds.), Advances in the Psychology of Thinking (pp. 1-44) London: Harvester- Wheatsheaf.

Ohlsson, S. (2011). Deep Learning: How the Mind Overrides Experience. Cambridge: Cambridge University Press.

Ormerod, T. C., MacGregor, J. N., Chronicle, E. P., Dewald, A. D., \& Chu, Y. (2013). Act first, think later: The presence and absence of inferential planning in problem solving. Memory \& Cognition, 41, 1096-1108.

Repovš, G., \& Baddeley, A. (2006). The multicomponent model of working memory: Explorations in experimental cognitive psychology. Neuroscience, 139, 5-21.

Schooler, J. W., \& Melcher, J. (1995). The ineffability of insight. In S. M. Smith, T. B. Ward, \& R. A. Finke (Eds.), The Creative Cognition Approach (pp. 97-133). Cambridge, MA: MIT Press. 
Schooler, J. W., Ohlsson, S., \& Brooks, K. (1993). Thoughts beyond words: When language overshadows insight. Journal of Experimental Psychology: General, 122, 166-183.

Sieck, W. R., Quinn, C. N., \& Schooler, J. W. (1999). Justification effects on the judgment of analogy. Memory \& Cognition 27, 844-855.

Siegler, R. S. (2000). Unconscious insights. Current Directions in Psychological Science, 9, 79-83.

Smith, R. W., \& Kounios, J. (1996). Sudden insight: All or none processing revealed by speed-accuracy decomposition. Journal of Experimental Psychology: Learning, Memory, \& Cognition, 22, 1443-1462.

Thompson, V. A., Prowse-Turner, J., Pennycook, G. R., Ball, L. J., Brack, H. M., Ophir, Y., \& Ackerman, R. (2013). The role of answer fluency and perceptual fluency as metacognitive cues for initiating analytic thinking. Cognition, 128, 237-251.

Weisberg, R. W. (2006). Creativity: Understanding Innovation in Problem Solving, Science, Invention, and the Arts. Hoboken, NJ: Wiley.

Weisberg, R. W. (this issue). Toward an integrated theory of insight in problem solving. Thinking \& Reasoning. 


\section{Footnotes}

${ }^{1}$ For an alternative theoretical view of the mechanisms underpinning articulatory suppression effects that raises serious questions about the involvement of phonological coding and storage see Jones, Hughes, and Macken $(2006,2007)$ and Jones, Macken, and Nicholls (2004). This alternative view, however, still maintains that articulatory suppression impairs the motor-planning involved in speech-based rehearsal such that opportunities for internalized, speech-based processing are severely reduced.

${ }^{2}$ An item-based survival analysis of the three insight problems revealed that the differences in the proportion of solvers over time across conditions was similar to the combined dataset for two out of the three problems, that is, the pigs in a pen problem and the triangle problem The anomalous problem was the deer problem, which revealed generally poor levels of performance in all conditions (i.e., the greatest level of success arose in the articulatory suppression condition, with $50 \%$ solvers). Because performance in the deer problem was generally deflated the item-based analysis for this problem revealed no reliable differences between conditions, $\chi^{2}(3, \mathrm{~N}=96)=2.61, p=.46$. 
Figure 1: Comparison of the proportions of problems solved across conditions over time, up to the maximum available problem solving time per task (i.e., 450 seconds). The text provides details of what each of the depicted data-points represents.

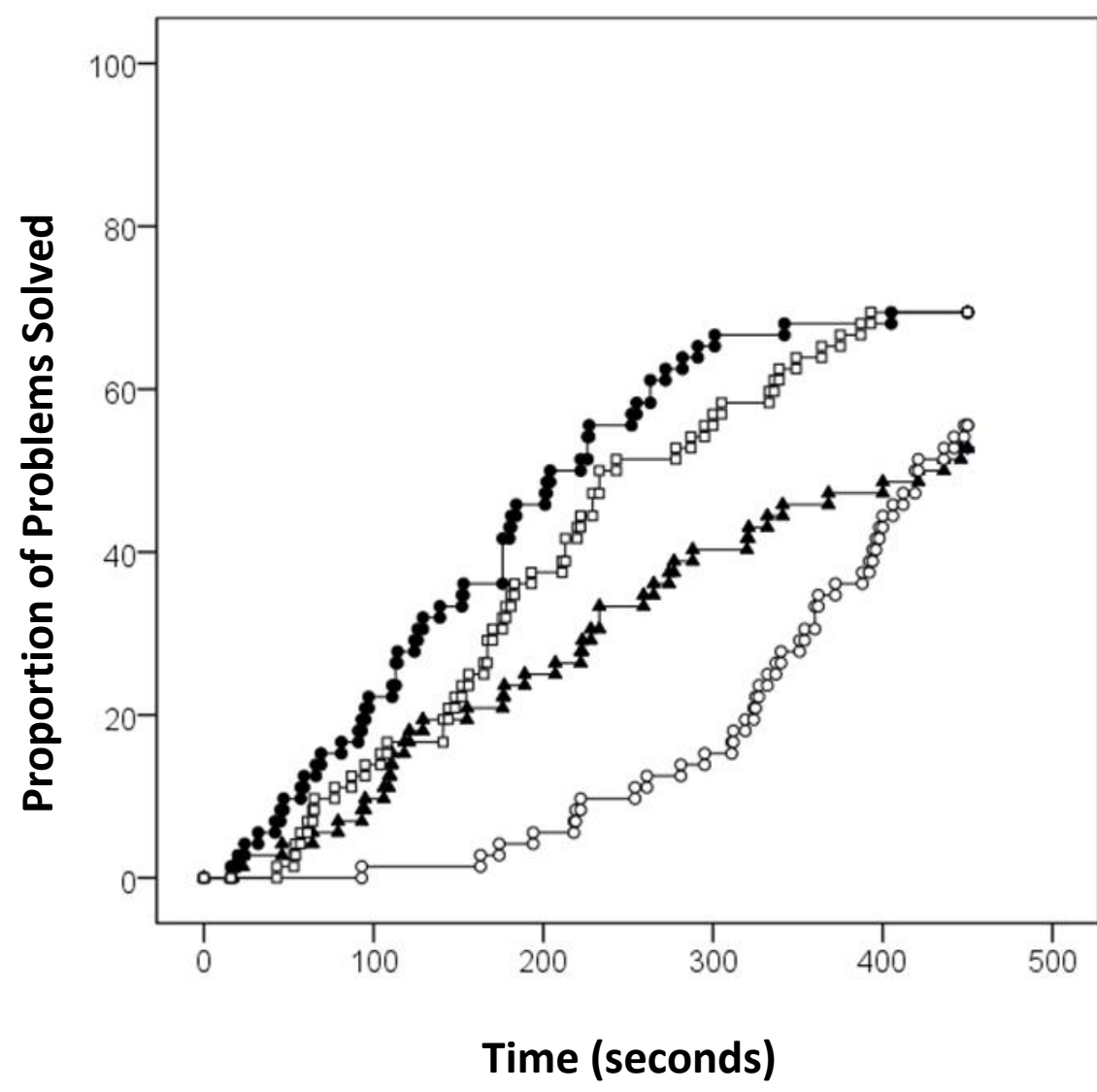

A Silent Working (Control)

Articulatory Suppression

$\square$ Irrelevant Speech

Thinking Aloud 
Figure 2: Comparison of the proportions of problems solved across conditions over time, up to the mid-point of the available problem solving time per task (i.e., 225 seconds).

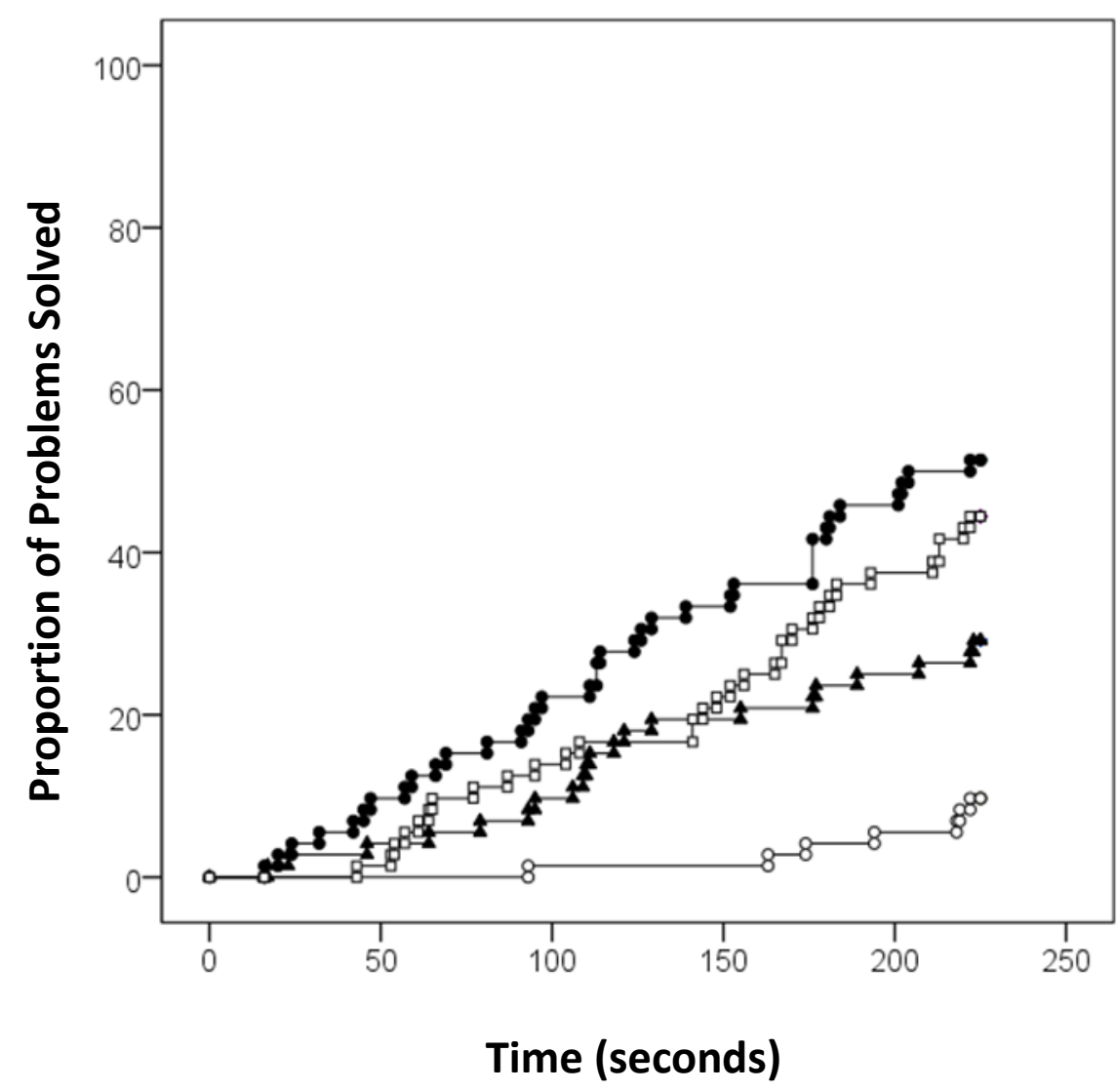

$\boldsymbol{\Delta}$ Silent Working (Control)

Articulatory Suppression

$\square$ Irrelevant Speech

Thinking Aloud 


\section{Appendix A}

\section{The Three Insight Problems Used in the Experiment}

The pigs in a pen problem: The initial state is shown on the left, with nine pigs enclosed within a square pen. Participants are asked to construct two more square pens so as to ensure that each pig ends up in a pen of its own. The solution to this problem is shown on the right.
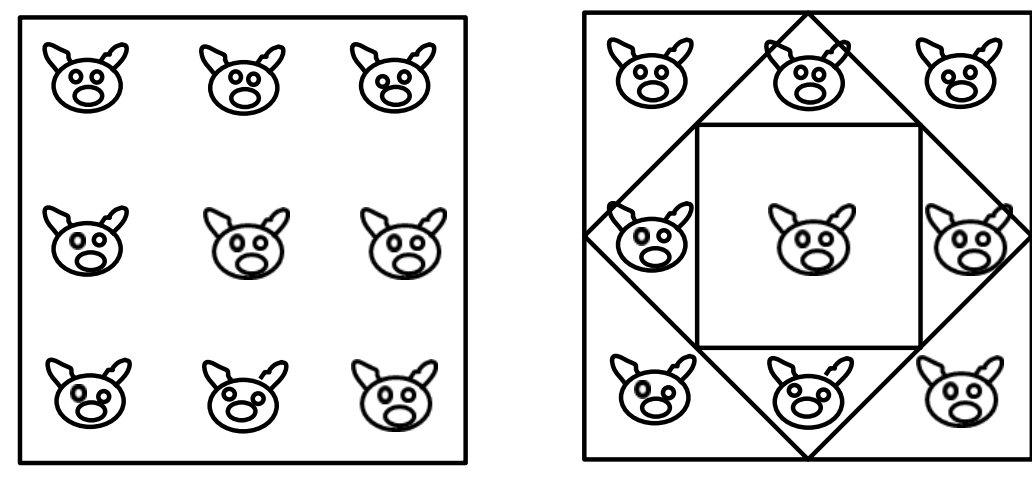

The triangle problem: The initial state is shown on the left, with the 10 identically-sized circles that form the shape of a triangle pointing towards the top of the page. Participants are asked to make the triangle shape point downwards by moving only three circles. The solution to this problem is shown on the right.
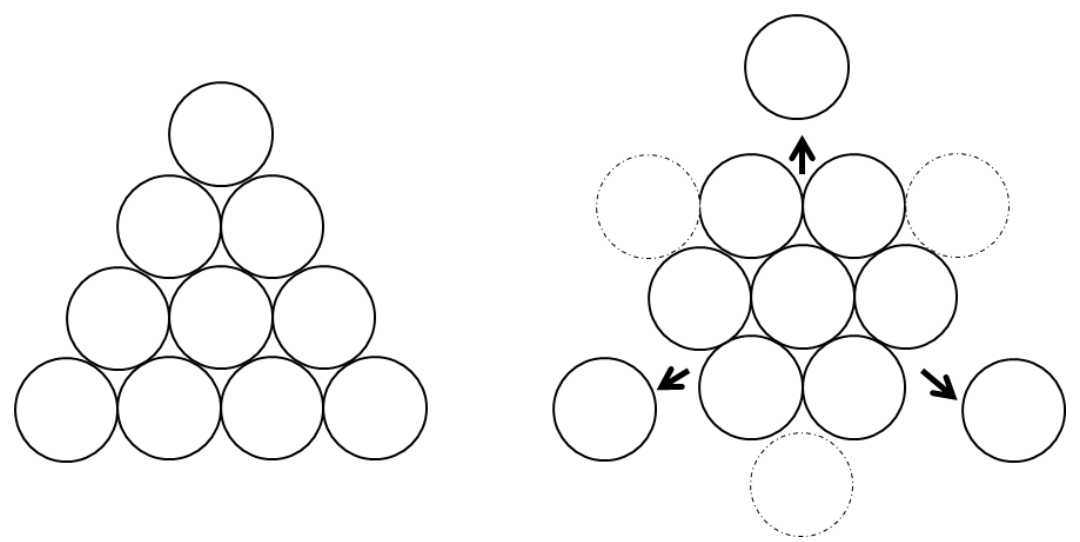
The deer problem: The initial state is shown on the left, with the deer being depicted using 5 lines of equal length. Participants are asked to make the deer face in a different direction by moving just one of lines. The solution to this problem is shown on the right.
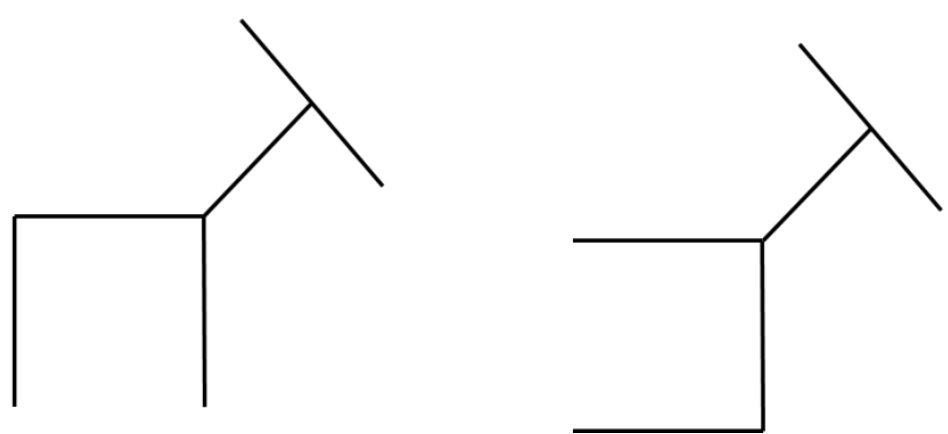